\title{
Research on Optimal Operation of Active Distribution Network with Energy Storage Facilities
}

\author{
Hua Wang ${ }^{1, *}$, Wenqi $\mathrm{Li}^{2}$, Haijing Yang ${ }^{1}$, Zhaohui $\mathrm{Li}^{1}$, and Tao $\mathrm{Shi}^{3}$ \\ ${ }^{1}$ State Grid Henan Electric Power Research Institute, 450052 Zhengzhou, China \\ ${ }^{2}$ State Grid Henan Electric Power Company, 450052 Zhengzhou, China \\ ${ }^{3}$ Nanjing University of Posts and Telecommunications, 210023 Nanjing, China
}

\begin{abstract}
With a large amount of intermittent distributed generation(DG) integrating into active distribution network(ADN), the flexibility requirements during the operation process should be paid more attention. Therefore, a dynamic optimal operation model is built. In this model, the maximization of economic benefits during the production cycle is set as the optimization objective, and the constrains of security and flexibility are considered. Then based on this model, the security, economy and flexibility requirements of power system are satisfied by coordination and interaction of different flexible sources. Finally, a case study is given to illustrate the validity and effectiveness of the models.
\end{abstract}

\section{Introduction}

An important task of power system operation is the optimization of generation scheduling and load allocation. According to difference of time scale, the optimal operation of power system includes static optimization and dynamic optimization [1-2]. Static optimization focus on the optimal objective at a certain moment. However, dynamic optimization focus on the multi-period optimization of generation sources based on the day-ahead dispatching framework. Dynamic optimization takes the coupling relationship of adjacent periods into account. Therefore, it is more suitable to the optimization of ADN operation [3-5].

For ADN, when it is an independent economic entity in a certain market environment, the ultimate objective of ADN operation is to make the benefit maximization under the precondition of system security and reliability. Usually, ADN operates under two business modes. In the first mode, ADN is a pure network operator, and only provide electricity distribution service. In the second mode, ADN is not only a network opera-tor, but also own some DGs as generation operator.

In this paper, a dynamic optimal operation model of ADN is built for the second business mode. The maximization of economic benefits during the production cycle is set as the optimization objective, and the constrains about security, flexibility etc. are considered. Then based on this model, coordination and interaction act between the network, generation and load, in order to meet of the security, economy and flexibility requirements [6-7].

\section{Objective of Optimal Operation Mode}

When ADN operate under the second business mode, the maximization of economic benefits during the production cycle is set as the optimization objective, as shown below:

$$
\max =\sum_{t=1}^{T}[R E(t)-C O(t)]
$$

Where, $T$ is the amount of time intervals; $R E(t)$ is the operation benefit of $\mathrm{ADN}$ at the t-th time interval; $C O(t)$ is the operation cost of $\mathrm{ADN}$ at the t-th time interval.

When the environment effectiveness is considered, the comprehensive benefit of ADN include two parts: one is the electricity benefit, the other is environment benefit. The electricity benefit depends on the amount of electricity sales and the corresponding electricity price. The environment benefit depends on the reduction amount of Carbon dioxide emission and the corresponding carbon-trade price. The reduction amount of Carbon dioxide emission is equal to the amount of Carbon dioxide discharged by coal-fired thermal power unit, which generated the same amount of electricity, as shown below:

$$
R E(t)=R E E(t)-R E C(t)
$$

Where, $R E E(t)$ is the electricity benefit of $\mathrm{ADN}$ at the t-th time interval; $R E C(t)$ is the environment benefit of $\mathrm{ADN}$ at the $\mathrm{t}$-th time interval.

The operation cost of ADN are comprised of electricity purchase cost, grid-loss cost, charge/discharge cost of energy storage(ES) facilities etc. as shown below:

$$
C O(t)=C O E(t)+C O L(t)+\operatorname{COS}(t)
$$

\footnotetext{
*Hua Wang: haiou8081@163.com.
} 
Where, $\operatorname{COE}(t)$ is the electricity purchase cost of $\mathrm{ADN}$ at the t-th time interval; $\operatorname{COL}(t)$ is the grid-loss cost of ADN at the t-th time interval; $\operatorname{COS}(t)$ is the charge/discharge cost of ES facilities at the t-th time interval.

\section{Equality Constrains of ADN Operation}

The equality constraints of ADN operation consider the factors such as benefit, cost, balance between power supply and demand.

\subsection{Electricity benefit}

The electricity benefit of ADN at the t-th time interval is shown as below:

$$
R E E(t)=T I N(t) \cdot L O(t) \cdot \Delta t
$$

Where, $\operatorname{TIN}(t)$ is the electricity sale price at the $\mathrm{t}$-th time interval; $L O(t)$ is the electricity sale price at the $\mathrm{t}$ th time interval; $\Delta t$ is time length of each interval.

\subsection{Environment benefit}

The environment benefit of ADN at the t-th time interval is shown as below:

$$
\begin{aligned}
& R E C(t)=C P \cdot E M C(t) \\
& E M C(t)=E R E(t) \cdot C E
\end{aligned}
$$

Where, $C P$ is the carbon-trade price; $E M C(t)$ is the reduction amount of Carbon dioxide emission at the $\mathrm{t}$-th time interval; $\operatorname{ERE}(t)$ is the amount of electricity produced by renewable generation source at the $t$-th time interval; $C E$ is the conversion coefficient between carbon emission and coal-fired by thermal power plant.

\subsection{Electricity purchase cost}

The electricity purchase cost of ADN at the t-th time interval is shown as below:

$$
\operatorname{COE}(t)=\sum_{i=1}^{N}[C P I(i) \cdot C P O(i, t) \cdot \Delta t]
$$

Where, $C P I(i)$ is electricity feed-in tariff of the $\mathrm{i}$-th DG; $C P O(i, t)$ is the feed-in power output of the i-th $\mathrm{DG}$ at the $\mathrm{t}$-th time interval.

\subsection{Grid-loss cost}

The grid-loss cost of ADN at the t-th time interval is shown as below:

$$
\operatorname{COL}(t)=\operatorname{TIN}(t) \cdot L O(t) \cdot \Delta t \cdot G L F
$$

Where, $\operatorname{TIN}(t)$ is electricity sales price of ADN at the t-th time interval; $G L F$ is the grid-loss coefficient, which is the proportion of grid-loss to total load.

\subsection{Charge/Discharge-loss cost of ES}

The charge/discharge-loss cost of ES at the t-th time interval is shown as below:

$$
\operatorname{COS}(t)=\operatorname{ESC} \cdot|\operatorname{ESP}(t)| \cdot \Delta t
$$

Where, $\operatorname{COS}(t)$ is charge/discharge-loss cost of ES at the t-th time interval; $\operatorname{ESP}(t)$ is charge/discharge power of $E S$ at the $t$-th time interval, if charge negative, else positive; ESC is loss cost of per unit charge/discharge electricity.

\subsection{Charge/Discharge energy balance of ES}

The charge/discharge energy balance of ES at the t-th time interval is shown as below:

$$
\operatorname{SOC}(t)=\operatorname{SOC}(t-1)-\operatorname{ESP}(t) \cdot \Delta t
$$

Where, $S O C(t)$ is energy status of ES at the $t$-th time interval; $\operatorname{SOC}(t-1)$ is energy status of ES at the $(\mathrm{t}-1)$-th time interval; $\operatorname{ESP}(t)$ is charge/discharge power of ES at the (t-1)-th time interval.

\subsection{Power balance of ADN}

The power balance of ADN at the t-th time interval is shown as below:

$$
\sum_{i=1}^{N} C S P(i, t)=L O(t) \cdot(1+G L F)
$$

Where, $\operatorname{CSP}(i, t)$ is active power output of the i-th DG at the t-th time interval; $L O(t)$ is to load supplied by $\mathrm{ADN}$ at the (t-1)-th time interval.

\section{Inequality Constrains of ADN}

When the grid structure has been determined, the operation of ADN should consider not only the grid transmission constraint, but also the constraints such as: upper and lower limitation of power output, flexible adjust ability, ramping ability, energy storage.

\subsection{Active power output constraint}

The active power output constraint of DG is shown as below:

$$
\min \_C S P(t) \leq C S P(i, t) \leq \max \_C S P(t)
$$

Where: $\operatorname{CSP}(i, t)$ is active power of the i-th DG at the $\mathrm{t}$-th time interval; $\min \_\operatorname{CSP}(t)$ is minimal active power of the $\mathrm{i}$-th $\mathrm{DG}$ at the $\mathrm{t}$-th time interval; max_CSP $(t)$ is maximal active power of the $\mathrm{i}$-th DG at the $\mathrm{t}$-th time interval.

For the dispatch DG, the active power output is affected by rated capacity, minimal technical output, etc. for the intermittent DG such as wind power generation, photovoltaic(PV) power generation, all the electricity produced by the these DGs are fully accommodated by 
ADN. For micro-grid(MG), the exchanging power between $\mathrm{MG}$ and main network should be maintained below a certain proportion of MG's rated capacity.

\subsection{Flexibility Constraint}

The flexibility constraint is to solve the problem caused by some uncertainty conditions, such as: change of solar irradiation, emergency, disturbance. The flexibility of ADN is depended not only on the inherent property of ADN, but also on the current operation status. Here, the flexibility constraint of ADN is shown as below [8-10]:

$$
\begin{aligned}
& \text { flexi }(t)_{+/-} \geq \text {flexi_re }(t)_{+/-} \\
& \text {flexi_re }(t)_{+/-}=\left[\text {flexi_capacity }(t), t_{\max }\right]
\end{aligned}
$$

Where, flex_re(t) $)_{+/-}$is the flexibility requirements of $\mathrm{ADN}$ at the t-th time interval, "+"means upward adjustment; “-"means downward adjustment; flexi_capacity $(t)$ is the flexible reserve capacity requirement of $\mathrm{ADN}$ at the $\mathrm{t}$-th time interval; $t_{\max }$ is the maximum of adjustment time length. The flexible coefficient is defined as shown below:

$$
\text { flexi_cf }=\frac{\text { flexi_capacity }(t)_{+/-}}{L O(t)}
$$

Where, flex_capacity $(t)_{+/-}$is the flexible reserve capacity of ADN at the t-th time interval; $L O(t)$ is the load power of ADN at the t-th time interval.

\subsection{Ramp-rate Constraint}

The dispatchable DGs should meet the ramp-rate constraint during the adjustment process, and include upward adjustment and downward adjustment, as shown below:

$$
|\operatorname{CSP}(i, t)-\operatorname{CSP}(i, t-1)| \leq r_{i} \cdot t_{\max }
$$

Where, $\operatorname{CSP}(i, t)$ is the active power output of the $\mathrm{i}$ th DG at the t-th time interval; $\operatorname{CSP}(i, t-1)$ is the active power output of the $\mathrm{i}$-th $\mathrm{DG}$ at the $(\mathrm{t}-1)$-th time interval; $r_{i}$ is ramp-rate of the $\mathrm{i}$-th $\mathrm{DG} ; t_{\max }$ is the allowable maximal adjustment time.

\subsection{Energy Storage Constraint}

The energy status of ES should be in the rated energy range, shown as below:

$$
\min \_S O C \leq S O C(t) \leq \max \_S O C
$$

Where, max_SOC is upper limitation of energy status; min_SOC is lower limitation of energy status; $\operatorname{SOC}(t)$ is the energy status of ES at the t-th time interval, usually between $20 \% \sim 80 \%$ of the rated energy.

\section{Case Study}

A typical daily scenario of ADN is used for case study. In which, the maximal load is $438 \mathrm{MW}$; the minimal load is $223 \mathrm{MW}$; the installed capacity of dispatchable DGs is 50MW; the installed capacity of distributed PV is 100MW; the installed capacity of distributed wind generation(WG) is $40 \mathrm{MW}$; the installed $\mathrm{ES}$ is $50 \mathrm{MW} / 100 \mathrm{MWh}$; the injection power from external network (EN) is $420 \mathrm{MW}$. The feed-in electricity price of ADN is $0.35 ¥ / \mathrm{kWh}$ between $1: 00-8: 00$ and is $0.6 ¥ / \mathrm{kWh}$

\begin{tabular}{|c|c|c|c|}
\hline No. & Load & WG & PV \\
\hline 1 & 319 & 40 & 0 \\
\hline 2 & 277 & 32 & 0 \\
\hline 3 & 252 & 34 & 0 \\
\hline 4 & 235 & 36 & 0 \\
\hline 5 & 227 & 30 & 0 \\
\hline 6 & 224 & 40 & 0 \\
\hline 7 & 223 & 20 & 0 \\
\hline 8 & 231 & 22 & 0 \\
\hline 9 & 250 & 16 & 15 \\
\hline 10 & 282 & 24 & 60 \\
\hline 11 & 325 & 22 & 72 \\
\hline 12 & 343 & 32 & 75 \\
\hline 13 & 345 & 26 & 110 \\
\hline 14 & 342 & 35 & 103 \\
\hline 15 & 334 & 26 & 113 \\
\hline 16 & 335 & 24 & 105 \\
\hline 17 & 337 & 20 & 75 \\
\hline 18 & 338 & 28 & 30 \\
\hline 19 & 347 & 26 & 15 \\
\hline 20 & 384 & 18 & 0 \\
\hline 21 & 438 & 24 & 0 \\
\hline 22 & 431 & 22 & 0 \\
\hline 23 & 408 & 26 & 0 \\
\hline 24 & 360 & 30 & 0 \\
\hline
\end{tabular}
between 9:00-24:00. The electricity sales price of EN is 0.35 $¥ / \mathrm{kWh}$ between 1:00-8:00 and is $0.46 ¥ / \mathrm{kWh}$ between 9:0024:00.

Table 1. The time series power of load and DGs (MW)

The property parameters of different DGs are shown in Table 2. Although the installed capacity of $M G$ is $60 \mathrm{MW}$, the feed-in power is limited to be lower than $20 \%$ of the rated capacity. For ES, the upper/lower limitation of active power output are $\pm 50 \mathrm{MW}$; the upper/lower limitation of SOC are $80 / 20 \mathrm{MWh}$; the ramp rate is $25 \mathrm{MW} / \mathrm{min}$; the feed-in electricity price is set equal to energy loss cost: $0.05 ¥ / \mathrm{kWh}$; the initial value of ES energy status is $20 \mathrm{MWh}$. For ADN, the grid-loss coefficient is 0.05 ; the flexible reserve capacity is 0.1 ; the maximum adjustment time is $5 \mathrm{~min}$. All the model and algorithm are programed on GAMS software. The optimal results for ADN operation are shown as Table 3.

Table2. Property parameter of dispatchable DGs

\begin{tabular}{|c|c|c|c|}
\hline Type & DG & MG & EN \\
\hline Rated Capacity (MW) & 50 & 60 & 420 \\
\hline Upper Limit (MW) & 50 & 12 & 420 \\
\hline Lower Limit (MW) & 0 & -12 & 0 \\
\hline
\end{tabular}




\begin{tabular}{|c|c|c|c|}
\hline Ramp Rate $(\mathrm{MW} / \mathrm{min})$ & 10 & 12 & 20 \\
\hline Electricity Price $(¥ / \mathrm{kWh})$ & 0.42 & 0.42 & TOU Price \\
\hline
\end{tabular}

Table3. Active power output of dispatchable DGs and EN.(MW)

\begin{tabular}{|c|c|c|c|c|}
\hline No & DG & MG & ES & EN \\
\hline 1 & 0 & 0 & 0 & 294.95 \\
\hline 2 & 0 & 0 & -10 & 268.85 \\
\hline 3 & 0 & 0 & -50 & 280.6 \\
\hline 4 & 0 & 0 & 0 & 210.75 \\
\hline 5 & 0 & 0 & 0 & 208.35 \\
\hline 6 & 0 & 0 & 0 & 195.2 \\
\hline 7 & 0 & 0 & 0 & 214.15 \\
\hline 8 & 0 & 0 & 0 & 220.55 \\
\hline 9 & 0 & 0 & 0 & 169.5 \\
\hline 10 & 50 & 12 & 0 & 150.1 \\
\hline 11 & 50 & 12 & 0 & 185.25 \\
\hline 12 & 50 & 12 & 0 & 191.15 \\
\hline 13 & 50 & 12 & 0 & 164.25 \\
\hline 14 & 50 & 12 & 50 & 109.1 \\
\hline 15 & 50 & 12 & 10 & 139.7 \\
\hline 16 & 50 & 12 & 0 & 160.75 \\
\hline 17 & 50 & 12 & 0 & 196.85 \\
\hline 18 & 50 & 12 & 0 & 234.9 \\
\hline 19 & 50 & 12 & 0 & 261.35 \\
\hline 20 & 50 & 12 & 0 & 323.2 \\
\hline 21 & 50 & 12 & 0 & 373.9 \\
\hline 22 & 50 & 12 & 0 & 368.55 \\
\hline 23 & 0 & 0 & 0 & 402.4 \\
\hline 24 & 0 & 0 & 0 & 348 \\
\hline
\end{tabular}

The results are shown in table 2. During the 1th interval to the 8 th interval and the 23rd interval to 24 th interval, the feed-in electricity price of dispatchable DGs is higher than the wholes ale price of EN, so the ADN firstly purchase electricity from EN. When during the 9th interval to the 22nd interval. the feed-in electricity price of dispatchable DGs is lower than the wholesale price of EN, so the ADN firstly purchase electricity from DG and MG. Through the optimization of whole process, the ADN get the maximal benefit: $432460 ¥$.

\section{Conclusions}

Along with a lot of intermittent DGs integrated into $\mathrm{ADN}$, the flexibility problem has aroused more and more concern. Flexible adjust sources such as ES, MG and other dispatchable DGs, are widely applied in ADN, in order to meet the flexibility requirement brought by intermittent DGs, such as PV and WG.

Then a dynamic optimal operation model of ADN is built in this paper. The maximization of economic benefits during the production cycle is set as the optimization objective, and the constrains about security, flexibility etc. are considered. Then the case study of ADN optimal operation is done based on a typical daily scenario. The security, economy and flexibility requirements of power system are satisfied by coordination and interaction of different flexible sources.

This work is supported by science and technology project of state grid corporation of China: research and application demonstration of the aggregation effect of multipoint distributed energy storage in power grid.

\section{References}

1. J.M. Guerrero, M. Chandorkar, T. Lee, IEEE Trans on Industrial Electronics, 60,1254(2013)

2. J.M. Guerrero, P.C. Loh, T.L. Lee, IEEE Trans on Industrial Electronics, 60,1263(2013)

3. C.L.T Borges, V.F. Martins, International Journal of Electrical Power\&Energy Systems, 26, 107(2012)

4. M. Fan, A. Su, Z.A. Zhang, The 21 st ICED Meeting, 21,4(2011)

5. S.S.A. Kaabi, H.H. Zeineldin, V. Khadkikar, IEEE Trans on Power Systems, 99,8(2014)

6. A. Rosso, J. Ma, D.S. Kirschen, 2011 50th IEEE CDC-ECC Meeting, 50,4361(2011)

7. J.H. Kehler, M. Hu, 2011 IEEE PES General Meeting, 47,3(2011)

8. N. Marco, Energy Policy , 38,7257(2010)

9. E. Lannoye, D. Flynn, M.O. Malley, 2011 IEEE PES General Meeting, 47,6(2011).

10. E. Lannoye, D. Flynn, M.O. Malley, IEEE Trans on Power Systems, 30,57(2015) 